\title{
Malignant Parathyroid Gland Neoplasm
}

National Cancer Institute

\section{Source}

National Cancer Institute. Malignant Parathyroid Gland Neoplasm. NCI Thesaurus. Code C9322.

A primary or metastatic malignant neoplasm affecting the parathyroid glands. 